University of Nebraska - Lincoln

DigitalCommons@University of Nebraska - Lincoln

M. Eugene Rudd Publications

Research Papers in Physics and Astronomy

$4-1981$

Ionization by Low and Intermediate Energy Ion and Neutral Beams

M. Eugene Rudd

University of Nebraska - Lincoln, erudd@unl.edu

Follow this and additional works at: https://digitalcommons.unl.edu/physicsrudd

Part of the Physics Commons

Rudd, M. Eugene, "Ionization by Low and Intermediate Energy lon and Neutral Beams" (1981). M. Eugene Rudd Publications. 2.

https://digitalcommons.unl.edu/physicsrudd/2

This Article is brought to you for free and open access by the Research Papers in Physics and Astronomy at DigitalCommons@University of Nebraska - Lincoln. It has been accepted for inclusion in M. Eugene Rudd Publications by an authorized administrator of DigitalCommons@University of Nebraska - Lincoln. 


\section{E. Rudd}

Behlen Laboratory of Physics

University of Nebraska-Lincoln Lincoln, NE 68588

\section{Introduct ion}

One of the most important basic processes that take place in collisions of atomic systems is ionization resulting in the emission of electrons. A complete understanding of this process is complicated by the fact that electron emission can take place via a number of mechanisms, some of which are related. Simple measurements of the cross section for electron ejection as a function of projectile velocity does not usually provide enough information to unravel the various mechanisms. But cross sections which are differential in the angle and energy of the ejected electrons, which are now available for protons on a variety of target gases, ${ }^{1}$ can be used to elucidate these mechanisms. While most of the experiments of this type have been done at high energies (above, say, $100 \mathrm{keV}$ ) there is now data available at energies as low as $5 \mathrm{keV}$ for protons on various gases ${ }^{2}$ and to $15 \mathrm{keV}$ for neutral hydrogen atoms on helium gas. ${ }^{3}$ At these low energies the angular distributions tend to become less anisotropic than at higher energies and do not yield as much information, but from the energy distributions integrated over angle we have been able to construct a mechanism which describes the results quite well. Also we have found a simple empirical equation for the cross sections differential in ejected electron energy which is of great potential usefulness to workers in various applied areas in which secondary electrons from collisions are involved.

\section{Experiment al Apparatus}

A beam of protons from the accelerator passes through the target gas at less than 1 mTorr pressure and is caught in a Faraday cup and integrated. Electrons from a short length of the beam path enter an electrostat ic analyzer and are detected by an electron multiplier and counted by a multiscaler. The angle between the beam and the analyzer-detector system can be varied from $10^{\circ}$ to $160^{\circ}$ and electrons down to about $2 \mathrm{eV}$ can be detected and counted with proper annulling of the earth's magnet ic field by Helmholtz coils and reduction of stray electric fields in the collision chamber.

For neutral impact a charge transfer cell with gas at several mTorr pressure is used to neutralize a fraction of the proton beam. A set of deflection plates removes the charged component from the beam leaving only neutrals to enter the chamber. The Faraday cup is replaced by a thermal detector, the emf of which is proportional to the beam current at any given energy. From the known geometry, the target gas pressure, and the number of electron counts for a given total number of beam particles, one can calculate the cross sections differential in the angle and energy of the ejected electrons. These doubly differential cross sections can be integrated in various ways to yield singly differential, total electron ejection cross sections, and average ejected electron energies.

\section{Low Energy Results for Protons}

Fig. I shows the cross sections, integrated over all angles, for electron ejection as a function of the eject ion energy $E$. The cross sections drop off $r$ apidly with $E$ and cover 4-5 orders of magnitude. Above $50 \mathrm{keV}$ one can see the beginning of the binary encounter peak which becomes much more prominent at st $i l l$

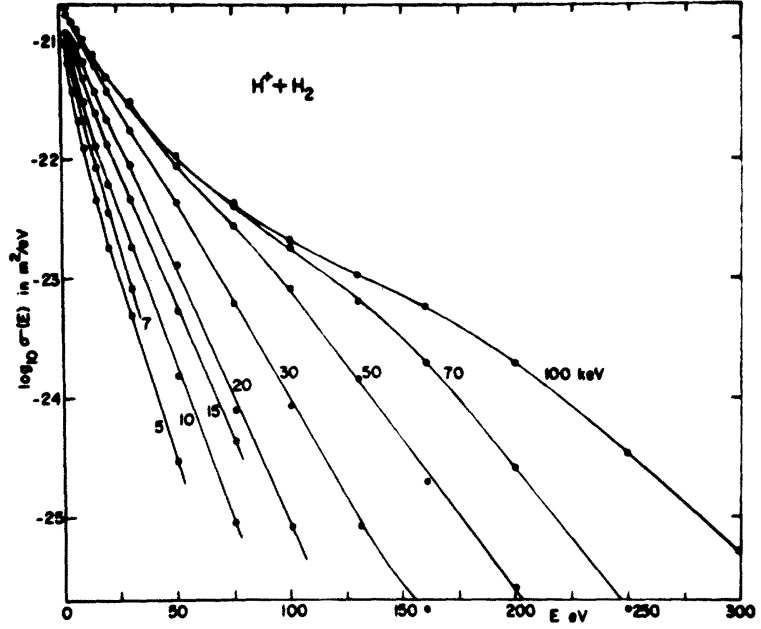

Figure 1. Cross sections for electron ejection from hydrogen gas by proton impact of various energies integrated over all angles.

higher impact energies. But this peak becomes less prominent at low energies and merges with the softcollision peak near zero energy to yield a monotomic straight line decrease in cross section with $E$. The nearly straight line behavior led to a search for an empirical equation describing the cross sections. The logarithmic slope of the line was found to follow an $E_{p}^{\frac{1}{2}}$ dependence where $E_{p}$ is the projectile energy. By comparing various gases it was also possible to see an $I^{-\frac{1}{2}}$ dependence where $I$ is the ionization potential of the target gas. The shape of the curves could be fit well by the expression exp $-\alpha E /(I T)^{\frac{1}{2}}$ where $T$ is the energy of an electron with the same velocity as the projectile and $\alpha$ is a dimensionless constant not much different from unity. Going further, it was possible to find an equation which gives the absolute values of the cross sections for the six different target gases for which data are available. This empirical equation uses two semiadjustable dimensionless parameters, both near unity. They are adjustable only in the sense that all gases tested fall into one of two categories, each with its set of parameters. The equation is

$$
\sigma(E)=5 \pi a_{0}^{2} \alpha{ }_{i}^{3} \frac{N_{i} I_{H}^{2}}{I_{i}^{3}} \frac{\left(T / I_{i}\right)^{\beta-\frac{1}{2}}}{4+\left(T / I_{i}\right)^{2 \beta}} \exp \left(-\frac{\alpha E}{\left(I_{i} T\right)^{\frac{1}{2}}}\right)
$$

where for $\mathrm{H}_{2}$ and $\mathrm{He}$ targets $\alpha=1.28$ and $\beta=1.0$, while for $\mathrm{N}_{2}, \mathrm{O}_{2}$, Ne and $\mathrm{Ar} \alpha=0.91$ and $\beta=0.75 . \mathrm{N}_{i}$ is the number of electrons in any orbital with binding energy $I_{i}, a_{0}$ is the Bohr radius, and $I_{H}=13.6 \mathrm{eV}$.

The partial cross section for the various atomic subshells are calculated separately and added. Figures 2 and 3 show how well the empirical equation fits. In Fig. 2 the ratio of the measured value to that calculated from eqn. (1) is plotted as a function of the electron energy (divided by the projectile energy) yielding a result not far from the ideal of unity for 


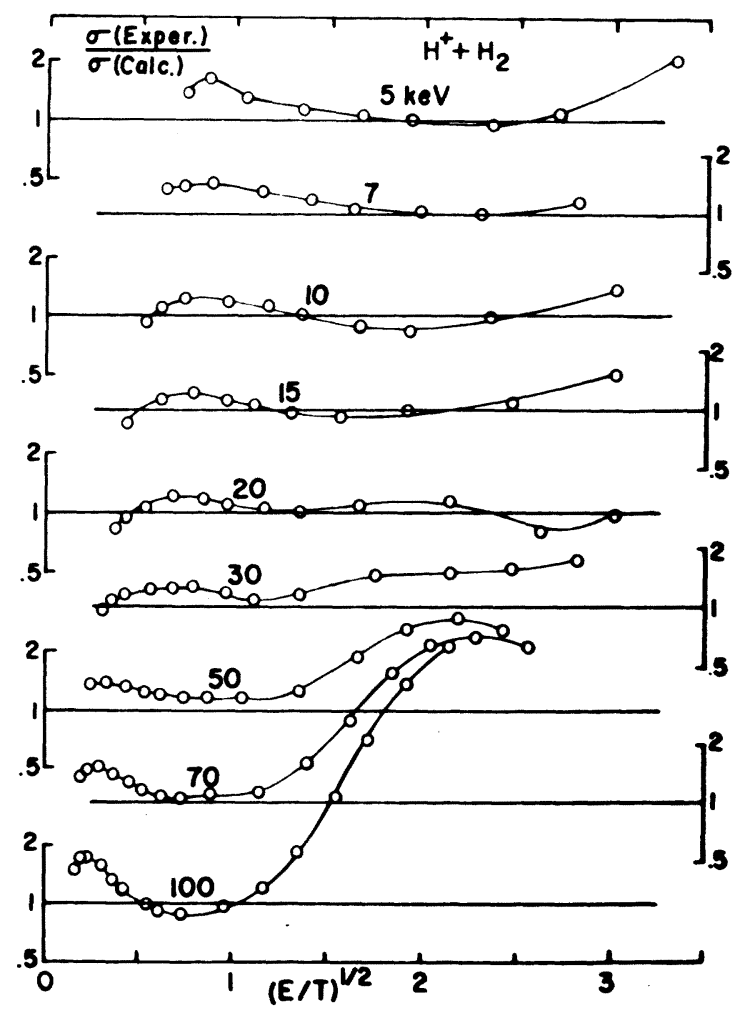

Figure 2. Rat io of experimental cross sections to those calculated by Eqn. I as a function of the ejected electron energy for various impact energies.

beam energies below about $50 \mathrm{keV}$. Above that energy the binary encounter peak begins to cause a large deviation. Fig. 3 shows the application of the equation

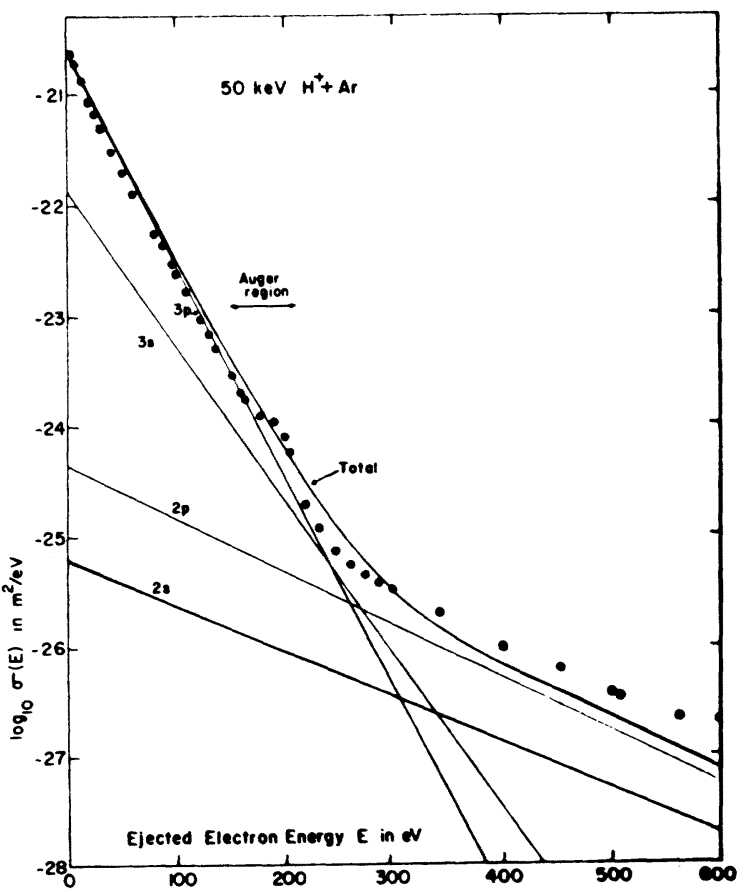

Figure 3. Cross sections for electron ejection from argon. Experimental values compared with those calculated from Eqn. I for each shell and added. to argon which has several subshells. It is apparent that while the loosely bound outer shell electrons contribute most to the cross section at low energies, the inner shells become dominant at high ejection energies. When the empirical equation is integrated over electron energies, the total cross section for electron ejection results.

$$
\sigma_{e}=5 \pi\left(a_{0} \alpha\right)^{2} \sum_{i} \frac{N_{i} I_{H}{ }^{2}}{I_{i}^{2}} \frac{\left(T / I_{i}\right)^{\beta}}{4+\left(T / I_{i}\right)^{2 \beta}}
$$

This equation fits known total cross section data quite well at all energies even though the differential cross section equation fails at high impact energy. The reason is that the binary encounter peak, though poorly described by eq (1) contributes very little to the integrated cross section.

\section{Theory}

The exponential dependence of the differential cross section on ejected electron energy can be derived from the molecular promotion model first suggested by Fano and Lichten. ${ }^{4}$ The derivation uses a result derived by Meyerhof ${ }^{5}$ for K-shell transitions. We are here applying it to all shells including outer shells. Fig. 4 shows a simplified correlation diagram. When

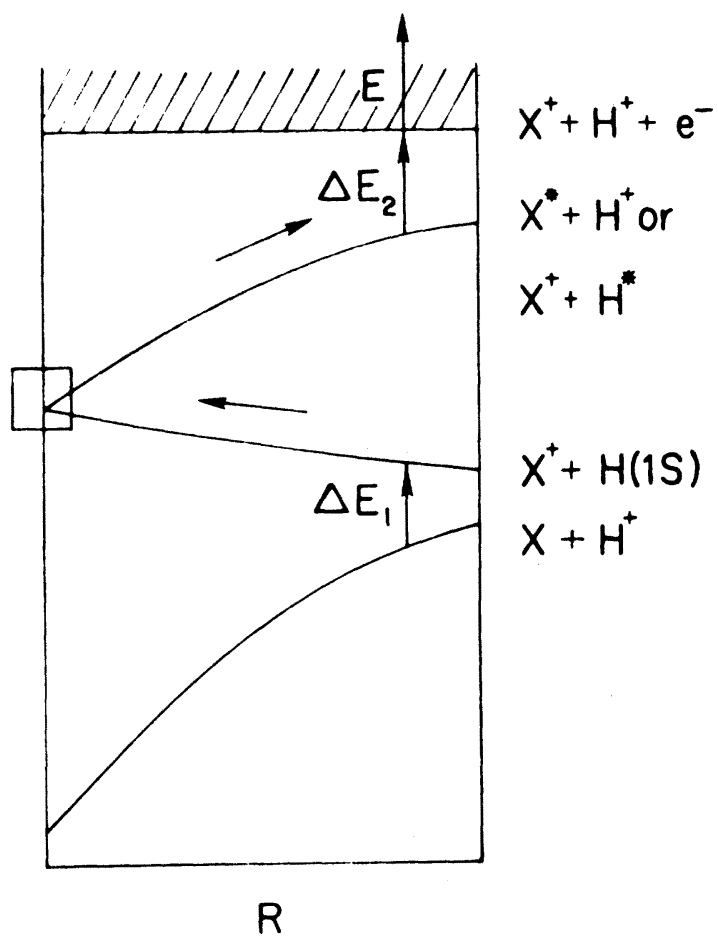

Figure 4. Simplified correlation diagram showing the transition proposed to account for the exponential energy distribution of ejected electrons.

the proton approaches the target $X$ a charge transfer can take place with an energy difference of $\Delta E_{1}$ and a probability $P_{1}$. Near the distance of closest approach a rotational coupling may promote the system to an excited state near the cont inuum. Then a transition to a cont inuum state with an energy difference of $\Delta E_{2}+E$ and probability $P_{2}$ results in a free electron of energy $E$. The cross section for the ent ire process can be written $\sigma(E)=P_{1} \sigma$ (rot) $P_{2}$. It is the factor $P_{2}$ that 
contains the energy $E$ which interests us. Meyerhof showed that $P_{2}=1 /\left(1+\exp 2 x_{2}\right)$ where $x_{2}=\left(E+\Delta E_{2}\right) l_{2} / 2 \hbar v_{p}$ where $v_{p}$ is the projectile velocity and $l_{2}$ is a characterist ic length. We take $I_{2}$ to be approximately equal to the diameter of the atom by setting $l_{2}=$ $2 \alpha a_{0}\left(I_{H} / I\right)^{\frac{1}{2}}$, where $\alpha$ is a dimensionless constant near unity which can be taken to be ident ical to the constant $\alpha$ in the empirical equation. Then if $T$ is small and $E$ not too small we can make the approximation that $\left(1+\exp 2 x_{2}\right)^{-1}=\exp -\Delta E_{2} /(I T)^{\frac{1}{2}} \exp -\alpha E /(I T)^{\frac{1}{2}}$ which is the desired exponential dependence on $E$.

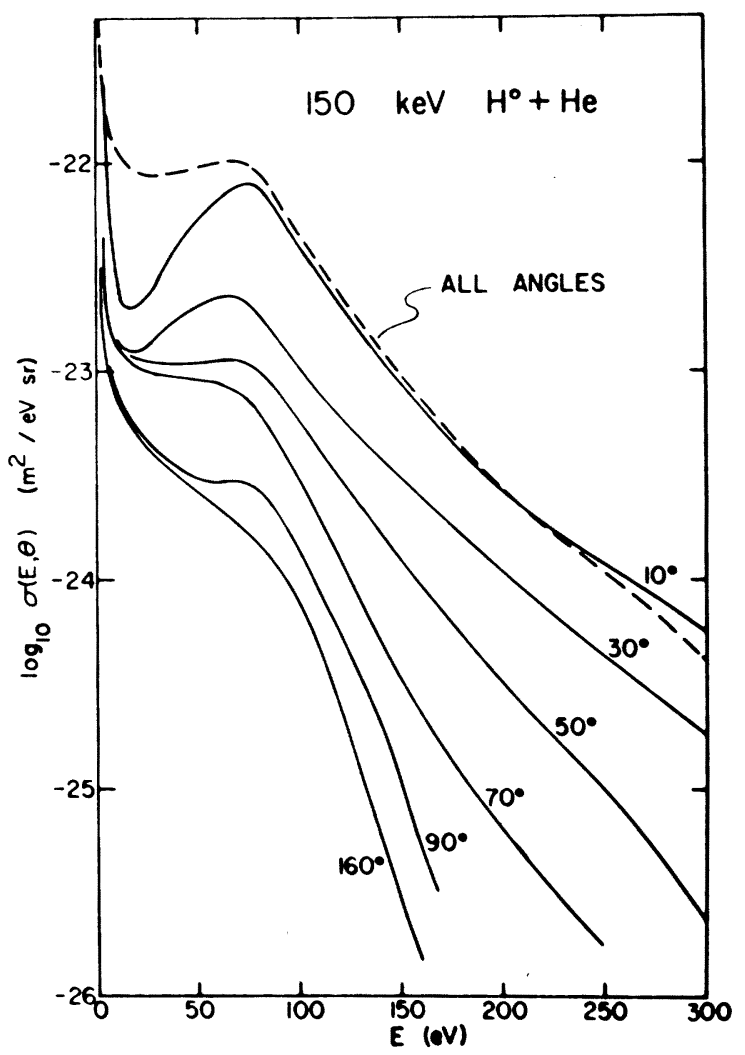

Figure 5. Energy distribution of electrons ejected at various angles by neutral hydrogen impacts on hel ium gas. Dotted line is for singly differential cross sections, integrated over all angles, in $\mathrm{m}^{2} / \mathrm{eV}$ on the same numerical scale.

\section{Results for Neutral Hydrogen Impact}

The main feature not iced in the energy distributions in Fig. 5 is a prominent peak at about $82 \mathrm{eV}$. This is the electron loss peak first noted by Wilson and Tobure $n^{6}$ for projectiles carrying electrons. Replotting the $10^{\circ}$ curve on a logarithmic energy scale, as in Fig. 6, we can make an interesting comparison with proton and electron impact data at the same incident velocity. At high ejected electron energies the proton and neutral cross sections are approximately the same since these electrons result from close collisions. At a lower energy where the velocity of the ejected electron equals that of the incident projec$t$ ile the neutral cross section is larger than the proton cross section due to the electron loss from the

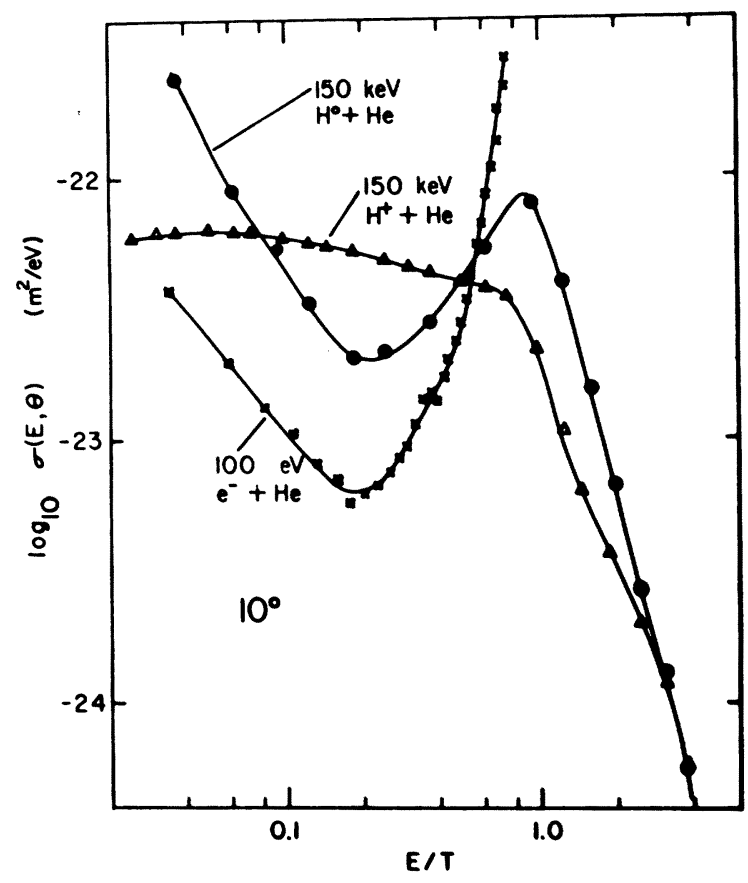

Figure 6. Comparison of electron energy distributions from hel ium gas bombarded by protons, neutral hydrogen, and electrons of approximately the same velocity.

projectile, as already noted. At still lower energies the electrons result from distant collisions. For these the screening of the nucleus by the electron in the project ile causes the neutral cross section to drop below that due to proton impact. At the lowest energies, however, the neutral cross section $r$ ises again to a larger value. We attribute this to target ionization by the electron carried by the neutral projectile.

Note that the shape of the distribution in that region is very similar to that from electrons of about the same velocity. It appears that for very distant collisions the orbital electron acts much like a free electron with the velocity of the projectile.

* This paper was prepared with the support of National Science Foundation Grant No. PHY79-05397.

\section{Acknowledgement}

The author wishes to thank Joseph Macek for suggesting the use of the molecular promotion model and helping work out the details.

\section{References}

1. See, e.g.,M.E. Rudd, L.H. Toburen, N. Stolterfoht, Atomic Data and Nuclear Data Tables 18, 413 (1976) and 23,405 (1979).

2. M.E. Rudd and D.H. Madison, Phys. Rev. A 15, 128 (1976), T.L. Criswell, L.H. Toburen and M.E. Rudd, Phys. Rev. A 16, 508 (1977), M.E. Rudd, Phys. Rev. A 20,787 (1979).

3. M.E. Rudd, J.S. Risley, J. Fryar, R.G. Rolfes, Phys. Rev. A 2l, 506 (1980).

4. U. Fano and W. Lichten, Phys. Rev. Letters 14, 627 (1965). 
5. W.E. Meyerhof, Phys. Rev. Letters 31, 1341 (1973).

6. W.E. Wilson and L.H. Toburen, Phys. Rev. A $\underline{7}$, 1535 (1973). 